\title{
Sarason's Conjecture of Toeplitz Operators on Fock-Sobolev Type Spaces
}

\author{
Xiaofeng Wang, ${ }^{1}$ Jianjun Chen $\mathbb{D}^{2},{ }^{2}$ and Jin Xia ${ }^{1}$ \\ ${ }^{1}$ School of Mathematics and Information Science and Key Laboratory of Mathematics and Interdisciplinary Sciences of the Guangdong \\ Higher Education Institute, Guangzhou University, Guangzhou 510006, China \\ ${ }^{2}$ School of Mathematics and Statistics, Zhaoqing University, Zhaoqing 526061, China
}

Correspondence should be addressed to Jianjun Chen; chenarmy@foxmail.com

Received 1 October 2017; Accepted 23 November 2017; Published 2 May 2018

Academic Editor: Aurelian Gheondea

Copyright (c) 2018 Xiaofeng Wang et al. This is an open access article distributed under the Creative Commons Attribution License, which permits unrestricted use, distribution, and reproduction in any medium, provided the original work is properly cited.

In this note, we will solve Sarason's conjecture on the Fock-Sobolev type spaces and give a well solution that if Toeplitz product $T_{u} T_{\bar{v}}$, with entire symbols $u$ and $v$, is bounded if and only if $u=e^{q}, v=C e^{-q}$, where $q$ is a linear complex polynomial and $C$ is a nonzero constant.

\section{Introduction}

Let $\mathbb{C}^{n}$ denote the complex $n$-space and $d v$ be the ordinary volume measure on $\mathbb{C}^{n}$ that is normalized so that $\int_{C^{n}} e^{-|z|^{2}} d v(z)=1$. If given any two points $z=\left(z_{1}, z_{2}, \ldots, z_{n}\right)$ and $w=\left(w_{1}, w_{2}, \ldots, w_{n}\right)$ in $\mathbb{C}^{n}$, we denote

$$
\begin{aligned}
z \cdot \bar{w} & =\sum_{j=1}^{n} z_{j} \overline{w_{j}}, \\
|z| & =\sqrt{z \cdot \bar{z}} .
\end{aligned}
$$

For every $0<p<\infty, \alpha \in \mathbb{R}$, we denote by $L_{\alpha}^{p}\left(\mathbb{C}^{n}\right)$ the space of measurable functions $f$ such that

$$
\|f\|_{L_{\alpha}^{p}}=\left(\int_{C^{n}}\left|f(z) e^{-(1 / 2)|z|^{2}}\right|^{p} \frac{d v(z)}{(1+|z|)^{\alpha}}\right)^{1 / p}<\infty .
$$

Let $H\left(\mathbb{C}^{n}\right)$ be the set of entire functions on $\mathbb{C}^{n}$. Then for a given $0<p<\infty$, the Fock-Sobolev type space $F_{\alpha}^{p}$ with the norm $\|\cdot\|_{F_{\alpha}^{p}}=\|\cdot\|_{L_{\alpha}^{p}}$ is defined as

$$
F_{\alpha}^{p}=\left\{f \in H\left(\mathbb{C}^{n}\right) \mid\|f\|_{L_{\alpha}^{p}}<\infty\right\} .
$$

Obviously, the Fock-Sobolev type space $F_{\alpha}^{2}$ equipped with the natural inner product defined by

$$
\langle f, g\rangle_{L_{\alpha}^{2}}=\int_{C^{n}} f(z) \overline{g(z)} e^{-|z|^{2}} \frac{d v(z)}{(1+|z|)^{\alpha}}
$$

is a reproducing kernel Hilbert space for every real $\alpha$. As stated in [1], with respect to the above inner product, it is difficult to compute the reproducing kernel of $F_{\alpha}^{2}$ explicitly. So we use the equivalent norm with respect to a new measure $|z|^{-\alpha} d v(z)$. In more detail, for $\alpha \leq 0$, we will let

$$
\langle f, g\rangle_{\alpha}=\int_{C^{n}} f(z) \overline{g(z)} e^{-|z|^{2}} \frac{d v(z)}{|z|^{\alpha}},
$$

and for $\alpha>0$ we let

$$
\begin{aligned}
\langle f, g\rangle_{\alpha}= & \int_{C^{n}} f_{\alpha / 2}^{-}(z) \overline{g_{\alpha / 2}^{-}(z)} e^{-|z|^{2}} d v(z) \\
& +\int_{C^{n}} f_{\alpha / 2}^{+}(z) \overline{g_{\alpha / 2}^{+}(z)} e^{-|z|^{2}} \frac{d v(z)}{|z|^{\alpha}},
\end{aligned}
$$

where $f_{\alpha / 2}^{-}$is the Taylor expansion of $f$ up to order $\alpha / 2$ and $f_{\alpha / 2}^{+}=f-f_{\alpha / 2}^{-}$. Now we can bravely make sure that the inner product $\langle\cdot, \cdot\rangle_{\alpha}$ generates a new Hilbert space norm on $F_{\alpha}^{2}$ that is equivalent to the $F_{\alpha}^{2}$ norm $\|\cdot\|_{F_{\alpha}^{2}}$. In particular, if we define the norm $\|\cdot\|_{\widetilde{F}_{\alpha}^{2}}$ on $F_{\alpha}^{2}$ by, when $\alpha \leq 0$,

$$
\|f\|_{\widetilde{F}_{\alpha}^{2}}=\left(\int_{C^{n}}|f(z)|^{2} e^{-|z|^{2}} \frac{d v(z)}{|z|^{\alpha}}\right)^{1 / 2}
$$


and when $\alpha>0$,

$$
\begin{aligned}
\|f\|_{\widetilde{F}_{\alpha}^{2}}= & \left(\int_{C^{n}}\left|(f)_{\alpha / 2}^{-}(z)\right|^{2} e^{-|z|^{2}} d v(z)\right)^{1 / 2} \\
& +\left(\int_{C^{n}}\left|(f)_{\alpha / 2}^{+}(z)\right|^{2} e^{-|z|^{2}} \frac{d v(z)}{|z|^{\alpha}}\right)^{1 / 2},
\end{aligned}
$$

and then we have that both $\|\cdot\|_{F_{\alpha}^{2}}$ and $\|\cdot\|_{F_{\alpha}^{2}}$ are equivalent norms.

As is well known, $F_{\alpha}^{2}$ is indeed a reproducing kernel Hilbert space (see Lemma 2.1 of [1] for more details). Therefore its reproducing kernel is

$$
K_{z}^{\alpha}(w)=\sum_{\beta} \phi_{\beta}(w) \overline{\phi_{\beta}(z)}
$$

where $\left\{\phi_{\beta}\right\}$ is any orthonormal basis for $F_{\alpha}^{2}$ with respect to $\langle\cdot, \cdot\rangle_{\alpha}$. Note that polynomials form a dense subset of $F_{\alpha}^{2}$ (see Proposition 2.3 in [2]). Also note that monomials are mutually orthogonal, which means that $\left\{z^{\beta} / \sqrt{\left\langle z^{\beta}, z^{\beta}\right\rangle_{\alpha}}\right\}$ is an orthonormal basis for $F_{\alpha}^{2}$. The arguments that are identical to the ones in the proof of Theorem 4.5 in [2] then give us that

$$
K_{z}^{\alpha}(w)= \begin{cases}\mathscr{I}^{-\alpha / 2} K_{z}(w), & \text { if } \alpha \leq 0 ; \\ \mathscr{I}^{-\alpha / 2} K_{z}(w)+\left(K_{z}\right)_{\alpha / 2}^{-}(w), & \text { if } \alpha>0 .\end{cases}
$$

Here $\mathscr{F}^{s}$ is the fractional integration operator defined as

$$
\mathscr{J}^{s} f(z)= \begin{cases}\sum_{k=0}^{\infty} \frac{\Gamma(n+k)}{\Gamma(n+s+k)} f_{k}(z), & \text { if } s \geq 0 ; \\ \sum_{k>|s|}^{\infty} \frac{\Gamma(n+k)}{\Gamma(n+s+k)} f_{k}(z), & \text { if } s<0,\end{cases}
$$

where each $f_{k}$ is a polynomial of degree $k$. Moreover, for $s>0$, $f_{s}^{+}$is the tail part of the Taylor expansion of $f$ of degree higher than $|s|$ given by

$$
f_{s}^{+}(z)=\sum_{k>|s|} f_{k}(z)
$$

and we let $f_{s}^{-}=f-f_{s}^{+}$(see [2] for more information on fractional differentiation and integration).

Now it is easy to see that if $\alpha \leq 0,\left(F_{\alpha}^{2},\|\cdot\|_{F_{\alpha}^{2}}\right)$ is a closed subspace of $L_{\alpha}^{2}$ with respect to $\langle\cdot, \cdot\rangle_{\alpha}$. In this case, let $P_{\alpha}$ denote the orthogonal projection, so that

$$
P_{\alpha} f(z)=\left\langle f, K_{z}^{\alpha}\right\rangle_{\alpha}
$$

for any $f \in L_{\alpha}^{2}$. Unfortunately, the inner product $\langle\cdot, \cdot\rangle_{\alpha}$ does not make sense on $L_{\alpha}^{2}$ when $\alpha>0$. That means we can not define the Toeplitz operator on $F_{\alpha}^{2}$ in the usual way in terms of this inner product. However according to the ideas of [1], it makes sense to define the Toeplitz operator with the symbols in $F_{\alpha}^{2}$ by the following formula:

$$
T_{\varphi}^{\alpha} f(z)=\int_{C^{n}} f(w) \varphi(w) \overline{K_{z}^{\alpha}(w)} e^{-|w|^{2}} \frac{d v(w)}{|w|^{\alpha}},
$$

if $\alpha \leq 0$ and

$$
\begin{aligned}
& T_{\varphi}^{\alpha} f(z) \\
& =\int_{C^{n}} \varphi(w)(f)_{\alpha / 2}^{-}(w) \overline{\left(K_{z}^{\alpha}\right)_{\alpha / 2}^{-}(w)} e^{-|w|^{2}} d v(w) \\
& \quad+\int_{C^{n}} \varphi(w)(f)_{\alpha / 2}^{+}(w) \overline{\left(K_{z}^{\alpha}\right)_{\alpha / 2}^{+}(w)} e^{-|w|^{2}} \frac{d v(w)}{|w|^{\alpha}}
\end{aligned}
$$

if $\alpha>0$, for any $\varphi, f \in F_{\alpha}^{2}$. In the sequel, we can reasonably define the Berezin transform of Toeplitz operator on $F_{\alpha}^{2}$ by

$$
\begin{aligned}
\widetilde{T_{\varphi}^{\alpha}}(z) & =\left\langle T_{\varphi}^{\alpha} k_{z}^{\alpha}, k_{z}^{\alpha}\right\rangle_{\alpha} \\
& =\int_{C^{n}} \varphi(w)\left|k_{z}^{\alpha}(w)\right|^{2} e^{-|w|^{2}} \frac{d v(w)}{|w|^{\alpha}}
\end{aligned}
$$

if $\alpha \leq 0$, and

$$
\begin{aligned}
\widetilde{T_{\varphi}^{\alpha}}(z)= & \left\langle T_{\varphi}^{\alpha} k_{z}^{\alpha}, k_{z}^{\alpha}\right\rangle_{\alpha} \\
= & \int_{C^{n}} \varphi(w)\left|\left(k_{z}^{\alpha}\right)_{\alpha / 2}^{-}(w)\right|^{2} e^{-|w|^{2}} d v(w) \\
& +\int_{C^{n}} \varphi(w)\left|\left(k_{z}^{\alpha}\right)_{\alpha / 2}^{+}(w)\right|^{2} e^{-|w|^{2}} \frac{d v(w)}{|w|^{\alpha}}
\end{aligned}
$$

if $\alpha>0$ for any $\varphi \in F_{\alpha}^{2}$, where $k_{z}^{\alpha}(w)$ is the normalization of the kernel $K_{z}^{\alpha}(w)$, that is, $k_{z}^{\alpha}(w)=K_{z}^{\alpha}(w) / \sqrt{K_{z}^{\alpha}(z)}$.

The original product problem, owed to Sarason firstly in [3], describes the pairs of outer functions $g$ and $h$ in the Hardy space such that the operator $T_{g} T_{\bar{h}}$ is bounded on the Hardy space. Sequentially, this problem was partially researched for the Hardy space in [4] and for the Bergman space in [5-8]. Unluckily it turns out that the Sarason's conjecture is not true for both Hardy space and Bergman space of unit disk. See $[9,10]$ for counterexamples.

We will, in this note, give the equivalent conditions about the Sarason's conjecture of Toeplitz product on Fock-Sobolev type spaces $F_{\alpha}^{2}$. Our main result will be the following.

Main Theorem. Suppose that $u$ and $v$ are two nonzero functions in Fock-Sobolev type spaces $F_{\alpha}^{2}$. Then the following conditions are equivalent:

(1) The Toeplitz product $T_{u}^{\alpha} T_{\bar{v}}^{\alpha}$ is bounded on Fock-Sobolev type spaces $F_{\alpha}^{2}$.

(2) There exists a complex linear polynomial $q(z)$ on $\mathbb{C}^{n}$ such that $u=e^{q}$ and $v=C e^{-q}$, where $C$ is a nonzero complex constant.

(3) The product $\widetilde{|u|^{2}} \widetilde{\left.v\right|^{2}}$ is a bounded function on the complex space $\mathbb{C}^{n}$.

In 2014, Cho et al. studied the products of Toeplitz operators on the classical Fock space (see [11]). In the case of the Fock-Sobolev space, Chen et al. (see [12]) had already proven the same topics and obtained the similar results. What is more, they claimed that if $f$ and $g$ are two nonzero 
functions in the Fock(-Sobolev) space, then the Toeplitz product $T_{f} T_{\bar{g}}$ is bounded if and only if $f=e^{q}$ and $g=C e^{-q}$, where $C$ is a nonzero constant and $q$ is a linear polynomial. More properties about Toeplitz operators on Fock-Sobolev spaces are referred to in [13]. Sequentially, Bommier-Hato et al. in [14] continued to research Cho's results on the general Fock-type space with the weight functions $\exp \left(-|\cdot|^{2 m}\right)$. They took full advantage of the exact form of the reproducing kernel of the general Fock-type space and concluded that if $u$ and $v$ are two nonzero functions, then the Toeplitz product $T_{u} T_{\bar{v}}$ is bounded if and only if $u=e^{g}$ and $v=C e^{-g}$, where $C$ is a nonzero constant and $g$ is a polynomial of degree at most $m$. The similar techniques are founded in $[15,16]$. However, the translations appearing to the classical Fock spaces are not suitable to the generalized Fock space. To tackle the main theorem, we have to use the main ideas of [14], that is, making good use of the explicit properties of the reproducing kernel $K_{z}^{\alpha}$ in Fock-Sobolev type spaces $F_{\alpha}^{2}$ instead of the Weyl operators defined by translations on the complex plane.

At last, it is remarked that, as stated in [1], the FockSobolev type spaces $F_{\alpha}^{2}$ are in fact very natural generalization of the Fock-Sobolev spaces and the Fock-Sobolev spaces of fractional order. For example, when $\alpha=0, F_{0}^{2}$ is the classical Fock space $F^{2}$. Thus in this paper, we always omit discussing the case of $\alpha=0$ and the similar result of this case is obtained in $[11,14]$.

Throughout this paper we write $X \leqslant Y$ or $Y \gtrsim X$ for nonnegative quantities $X$ and $Y$ whenever there is a constant $C>0$ independent of $X$ and $Y$ such that $X \leq C Y$. Similarly we write $X \simeq Y$ if $X \lesssim Y$ and $Y \lesssim X$.

\section{Proof of the Main Result}

We begin with some properties of the Fock-Sobolev spaces $F_{\alpha}^{2}$. See [1] for more information.

Lemma 1. Suppose that $f$ belongs to the Fock-Sobolev type space $F_{\alpha}^{p}$ for any real $\alpha$. Then for any $z, w \in \mathbb{C}^{n}$, we have

$$
|f(z)|^{p} e^{-(p / 2)|z|^{2}} \frac{1}{(1+|z|)^{\alpha}} \lesssim\|f\|_{F_{\alpha}^{p}}^{p},
$$

and when $\alpha<0$,

$$
\begin{aligned}
& \left|K_{z}^{\alpha}(w)\right| \\
& \lesssim(1+|z||w|)^{\alpha / 2} \exp \left(\frac{1}{2}|z|^{2}+\frac{1}{2}|w|^{2}-\frac{1}{8}|z-w|^{2}\right),
\end{aligned}
$$

when $\alpha>0$,

$$
\begin{aligned}
\left|K_{z}^{\alpha}(w)\right| \leq & (1+|w \cdot \bar{z}|)^{\alpha / 2} \\
& \cdot \exp \left(\frac{1}{2}|z|^{2}+\frac{1}{2}|w|^{2}-\frac{1}{8}|z-w|^{2}\right) .
\end{aligned}
$$

More specifically,

$$
\left|K_{z}^{\alpha}(z)\right| \simeq(1+|z|)^{\alpha} e^{|z|^{2}}
$$

for any $z \in \mathbb{C}^{n}$ and there is a $r>0$ such that

$$
\left|K_{z}^{\alpha}(w)\right| \gtrsim(1+|z|)^{\alpha} \exp \left(\frac{1}{2}|z|^{2}+\frac{1}{2}|w|^{2}\right),
$$

for any $z \in B(w, r)$.

A consequence of the first estimate in Lemma 1 is that, for any function $u \in F_{\alpha}^{2}$, the Toeplitz operators $T_{u}$ and $T_{\bar{u}}$ are both densely defined on $F_{\alpha}^{2}$.

Lemma 2. If the function $u$ belongs to the Fock-Sobolev type space $F_{\alpha}^{2}$, we then have $\left(T_{u}^{\alpha}\right)^{*}=T_{\bar{u}}^{\alpha}$.

Proof. In views of the Lemma 3.4 in [1], we can calculate that, for any polynomial $f$ and $g \in F^{\infty}$ (see [1] for the definition of $F^{\infty}$ ),

$$
\begin{aligned}
\left\langle\left(T_{u}^{\alpha}\right)^{*} f, g\right\rangle_{\alpha} & =\int_{C^{n}} \bar{u}(z) f(z) \overline{g(z)} \frac{e^{-|z|^{2}}}{|z|^{\alpha}} d v(z) \\
& =\left\langle T_{\bar{u}}^{\alpha} f, g\right\rangle_{\alpha},
\end{aligned}
$$

if $\alpha \leq 0$, and if $\alpha>0$,

$$
\begin{aligned}
\left\langle\left(T_{u}^{\alpha}\right)^{*} f, g\right\rangle_{\alpha}= & \int_{C^{n}} \bar{u}(z) f_{\alpha / 2}^{-}(z) \overline{g_{\alpha / 2}^{-}(z)} e^{-|z|^{2}} d v(z) \\
& +\int_{C^{n}} \bar{u}(z) f_{\alpha / 2}^{+}(z) \overline{g_{\alpha / 2}^{+}(z)} \frac{e^{-|z|^{2}}}{|z|^{\alpha}} d v .
\end{aligned}
$$

Lastly the fact that the set of all holomorphic polynomials is dense in $F_{\alpha}^{2}$ completes the proof.

Lemma 3. For given $u, v \in F_{\alpha}^{2}$, if $T_{u}^{\alpha} T_{\bar{v}}^{\alpha}$ is bounded on $F_{\alpha}^{2}$, then $T_{u}^{\alpha} T_{\bar{v}}^{\alpha} K_{z}^{\alpha}(w)=u(w) \bar{v}(z) K_{z}^{\alpha}(w)$ for any $z, w \in \mathbb{C}^{n}$.

Proof. When $\alpha \leq 0$, in view of reproducing properties of $K_{z}^{\alpha}(w)$, Lemma 3.4, the claim $(d) \Rightarrow(a)$ of Lemma 3.10 in [1], and Lemma 2, we see that

$$
\begin{aligned}
T_{u}^{\alpha} T_{\bar{v}}^{\alpha} K_{z}^{\alpha}(w) & =\left\langle T_{u}^{\alpha} T_{\bar{v}}^{\alpha} K_{z}^{\alpha}, K_{w}^{\alpha}\right\rangle_{\alpha} \\
& =\left\langle\bar{v}(z) K_{z}^{\alpha}, \bar{u}(w) K_{w}^{\alpha}\right\rangle_{\alpha} \\
& =u(w) \bar{v}(z) K_{z}^{\alpha}(w) .
\end{aligned}
$$

On the other side, when $\alpha>0$, we have to use the Lemma 3.4, the claim $(d) \Rightarrow(a)$ of Lemma 3.10 in [1] to achieve that, if $u, v \in F_{\alpha}^{2}, T_{u}^{\alpha} T_{\bar{v}}^{\alpha}$ is bounded,

$$
\begin{aligned}
& \left\langle T_{u}^{\alpha} T_{\bar{v}}^{\alpha} K_{z}^{\alpha}, K_{w}^{\alpha}\right\rangle_{\alpha} \\
& \quad=\int_{C^{n}} u(\lambda)\left(T_{\bar{v}}^{\alpha} K_{z}^{\alpha}\right)_{\alpha / 2}^{-}(\lambda) \overline{\left(K_{w}^{\alpha}\right)_{\alpha / 2}^{-}(\lambda)} e^{-|\lambda|^{2}} d v(\lambda) \\
& \quad+\int_{C^{n}} u(\lambda)\left(T_{\bar{v}}^{\alpha} K_{z}^{\alpha}\right)_{\alpha / 2}^{+}(\lambda) \overline{\left(K_{w}^{\alpha}\right)_{\alpha / 2}^{+}(\lambda)} \frac{e^{-|\lambda|^{2}}}{|\lambda|^{\alpha}} d v(\lambda) .
\end{aligned}
$$


Together with (3.5) in [1], Fubini's theorem, and the reproducing property, we can see that

$$
\begin{aligned}
& \int_{C^{n}} u(\lambda)\left(T_{\bar{v}}^{\alpha} K_{z}^{\alpha}\right)_{\alpha / 2}^{+}(\lambda) \overline{\left(K_{w}^{\alpha}\right)_{\alpha / 2}^{+}(\lambda)} \frac{e^{-|\lambda|^{2}}}{|\lambda|^{\alpha}} d v(\lambda) \\
& =\int_{C^{n}} \int_{C^{n}} u(\lambda) \bar{v}(\xi)\left(K_{z}^{\alpha}\right)_{\alpha / 2}^{+}(\xi)\left(K_{\xi}^{\alpha}\right)_{\alpha / 2}^{+}(\lambda) \\
& \overline{\left(K_{w}^{\alpha}\right)_{\alpha / 2}^{+}(\lambda)} \frac{e^{-|\lambda|^{2}}}{|\lambda|^{\alpha}} \frac{e^{-|\xi|^{2}}}{|\xi|^{\alpha}} d v(\xi) d v(\lambda) \\
& =\int_{C^{n}} u(\lambda)\left(K_{\xi}^{\alpha}\right)_{\alpha / 2}^{+}(\lambda) \overline{\left(K_{w}^{\alpha}\right)_{\alpha / 2}^{+}(\lambda)} \frac{e^{-|\lambda|^{2}}}{|\lambda|^{\alpha}} d v(\lambda) \\
& \cdot \int_{C^{n}} \bar{v}(\xi)\left(K_{z}^{\alpha}\right)_{\alpha / 2}^{+}(\xi) \frac{e^{-|\xi|^{2}}}{|\xi|^{\alpha}} d v(\xi)=u(w) \int_{C^{n}} \bar{v}(\xi) \\
& \cdot\left(K_{\xi}^{\alpha}\right)_{\alpha / 2}^{+}(w)\left(K_{z}^{\alpha}\right)_{\alpha / 2}^{+}(\xi) \frac{e^{-|\xi|^{2}}}{|\xi|^{\alpha}} d v(\xi)=u(w) \bar{v}(z) \\
& \cdot\left(K_{z}^{\alpha}\right)_{\alpha / 2}^{+}(w) .
\end{aligned}
$$

Similarly, we can achieve that

$$
\begin{aligned}
& \int_{C^{n}} u(\lambda)\left(T_{\bar{v}}^{\alpha} K_{z}^{\alpha}\right)_{\alpha / 2}^{-}(\lambda) \overline{\left(K_{w}^{\alpha}\right)_{\alpha / 2}^{-}(\lambda)} e^{-|\lambda|^{2}} d v(\lambda) \\
& =u(w) \bar{v}(z)\left(K_{z}^{\alpha}\right)_{\alpha / 2}^{-}(w) .
\end{aligned}
$$

Therefore,

$$
\begin{aligned}
T_{u}^{\alpha} T_{\bar{v}}^{\alpha} K_{z}^{\alpha}(w) & =\left\langle T_{u}^{\alpha} T_{\bar{v}}^{\alpha} K_{z}^{\alpha}, K_{w}^{\alpha}\right\rangle_{\alpha} \\
& =u(w) \bar{v}(z) K_{z}^{\alpha}(w) .
\end{aligned}
$$

Theorem $4((1) \Rightarrow(2))$. If we give that $u$ and $v$ are two nonzero functions in the Fock-Sobolev type space $F_{\alpha}^{2}$ such that Toeplitz product $T_{u}^{\alpha} T_{\bar{v}}^{\alpha}$ is bounded on $F_{\alpha}^{2}$; then there is a complex linear polynomial $q(z)$ on $\mathbb{C}^{n}$ such that $u=e^{q}$ and $v=C e^{-q}$, where $C$ is a nonzero complex constant.

Proof. This proof is similar to Theorem 2.4 in [12] and here we only give its brief illustration.

If the condition holds that the Toeplitz product $T_{u}^{\alpha} T_{\bar{v}}^{\alpha}$ is bounded on $F_{\alpha}^{2}$, by Lemmas 2 and 3 and the Cauchy-Schwarz inequality, we can see that

$$
|u(z) v(z)|=\left|\widetilde{T_{u}^{\alpha} T_{\bar{v}}^{\alpha}}\right| \leq\left\|T_{u}^{\alpha} T_{\bar{v}}^{\alpha}\right\|<\infty .
$$

Sequentially, the local property of reproducing kernel $K_{z}$ shows us that the module of function

$$
T(z, w)=\frac{\left\langle T_{u}^{\alpha} T_{\bar{v}}^{\alpha} K_{w}^{\alpha}, K_{z}^{\alpha}\right\rangle_{\alpha}}{\sqrt{K_{z}^{\alpha}(z)} \sqrt{K_{w}^{\alpha}(w)}}
$$

is equivalent to $\left|e^{q(z)-\overline{q(w)}}\right|$ when $|z-w|<\epsilon_{0}$. It implies that $|T(z, w)|$ is bounded in that situation.
On the other side, we give the representation of quadratic polynomial in the case of real inner product as follows: $q_{2}(z)=\langle A z, z\rangle$, where $q=q_{1}+q_{2}, q_{1}$ is linear, $q_{2}$ is a homogeneous polynomial of degree 2 , and $A=A_{n \times n}$ is a complex matrix symmetric in the real sense. After we choose $w=r \xi$ and $z=r \xi+\left(\epsilon_{0} / 2\right) \eta$, where $r$ is any real positive number, we achieve that

$$
\left|e^{q(z)-\overline{q(w)}}\right|=M \exp \left(r \epsilon_{0}\langle A \xi, \eta\rangle\right)
$$

is not bounded as $r \rightarrow \infty$. This contradiction finishes the proof.

Theorem $5((2) \Rightarrow(1))$. If $u=e^{q}$ and $v=e^{-q}$ where $q$ is a complex linear polynomial on $\mathbb{C}^{n}$, then $T_{u}^{\alpha} T_{\bar{v}}^{\alpha}$ is bounded on the Fock-Sobolev type space $F_{\alpha}^{2}$.

Proof. To prove the boundedness of $T_{u}^{\alpha} T_{\bar{v}}^{\alpha}$, we will sufficiently obtain that $\left\|T_{u}^{\alpha} T_{\bar{v}}^{\alpha} f\right\|_{F_{\alpha}^{2}}$ is bounded by means of the idea of [1]. In fact we only discuss the case of $\alpha>0$ because the other case is the same as the proof of Theorem 2.5 in [12]. Using the similar ways, our goal is to obtain that

$$
\begin{gathered}
\left\|T_{u}^{\alpha} T_{\bar{v}}^{\alpha} f\right\|_{\vec{F}_{\alpha}^{2}}=\left(\int_{C^{n}} \mid\left(T_{u}^{\alpha} T_{\bar{v}}^{\alpha} f\right)_{\alpha / 2}^{-}(z)\right. \\
\left.\left.\cdot e^{-(1 / 2)|z|^{2}}\right|^{2} d v(z)\right)^{1 / 2} \\
+\left(\int_{C^{n}} \mid\left(T_{u}^{\alpha} T_{\bar{v}}^{\alpha} f\right)_{\alpha / 2}^{+}(z)\right. \\
\left.\left.\cdot e^{-(1 / 2)|z|^{2}}|z|^{-\alpha / 2}\right|^{2} d v(z)\right)^{1 / 2}
\end{gathered}
$$

is bounded for any $f \in F_{\alpha}^{2}$ in view of the definition of the norm $\|\cdot\|_{\vec{F}_{\alpha}^{2}}$. To the end, we focus our attention on the integrands in it. By formulae (3.4) (3.5) in [1] and the definition of Toeplitz operator, the integrands in the norm are

$$
\begin{aligned}
& \left(T_{u}^{\alpha} T_{\bar{v}}^{\alpha} f\right)_{\alpha / 2}^{-}(z)=\int_{C^{n}} \bar{v}(\eta)(f)_{\alpha / 2}^{-}(\eta)\left(\int_{C^{n}} u(w)\right. \\
& \left.\cdot \overline{\left(K_{w}^{\alpha}\right)_{\alpha / 2}^{-}(\eta)\left(K_{z}^{\alpha}\right)_{\alpha / 2}^{-}(w)} e^{-|w|^{2}} d v(w)\right) e^{-|\eta|^{2}} d v(\eta), \\
& \left(T_{u}^{\alpha} T_{\bar{v}}^{\alpha} f\right)_{\alpha / 2}^{+}(z)=\int_{C^{n}} \bar{v}(\eta)(f)_{\alpha / 2}^{+}(\eta)\left(\int_{C^{n}} u(w)\right. \\
& \left.\cdot \frac{\left(K_{w}^{\alpha}\right)_{\alpha / 2}^{+}(\eta)\left(K_{z}^{\alpha}\right)_{\alpha / 2}^{+}(w)}{|w|^{\alpha}} d v(w)\right) \\
& \cdot \frac{e^{-|\eta|^{2}}}{|\eta|^{\alpha}} d v(\eta) .
\end{aligned}
$$

By the reproducing property, the estimations of their module are, respectively, coming from

$$
\begin{aligned}
& \left|\left(T_{u}^{\alpha} T_{\bar{v}}^{\alpha} f\right)_{\alpha / 2}^{-}(z)\right| \lesssim \int_{C^{n}}|u(z)||\bar{v}(\eta)|\left|(f)_{\alpha / 2}^{-}(\eta)\right| \\
& \cdot\left|\left(K_{z}^{\alpha}\right)_{\alpha / 2}^{-}(\eta)\right| e^{-|\eta|^{2}} d v(\eta),
\end{aligned}
$$


and then, similarly,

$$
\begin{aligned}
& \left|\left(T_{u}^{\alpha} T_{\bar{v}}^{\alpha} f\right)_{\alpha / 2}^{+}(z)\right| \lesssim \int_{C^{n}}|u(z)||\bar{v}(\eta)|\left|(f)_{\alpha / 2}^{+}(\eta)\right| \\
& \cdot\left|\left(K_{z}^{\alpha}\right)_{\alpha / 2}^{+}(\eta)\right| \frac{e^{-|\eta|^{2}}}{|\eta|^{\alpha}} d v(\eta) .
\end{aligned}
$$

Therefore, using the Cauchy-Schwarz inequality, we have the estimation of the first term of the norm as follows:

$$
\begin{gathered}
\int_{C^{n}}\left|\left(T_{u}^{\alpha} T_{\bar{v}}^{\alpha} f\right)_{\alpha / 2}^{-}(z) e^{-(1 / 2)|z|^{2}}\right|^{2} d v(z) \\
\leq \int_{C^{n}}\left(\int_{C^{n}} H_{\alpha}^{1}(w, z) d v(w) \int_{C^{n}} H_{\alpha}^{1}(w, z)\right. \\
\left.\cdot\left|(f)_{\alpha / 2}^{-}(w)\right|^{2} e^{-|w|^{2}} d v(w)\right) d v(z),
\end{gathered}
$$

where $H_{\alpha}^{1}(w, z)=e^{-(1 / 2)|z|^{2}}\left|\left(K_{z}^{\alpha}\right)_{\alpha / 2}^{-}(w)\right| e^{-(1 / 2)|w|^{2}} \exp (\operatorname{Re}(q(z)$ $-\overline{q(w)})$ ). Similarly the estimation of the second norm has been achieved that

$$
\begin{gathered}
\left.\left.\int_{C^{n}}\left|\left(T_{u}^{\alpha} T_{\bar{v}}^{\alpha} f\right)_{\alpha / 2}^{+}(z) e^{-(1 / 2)|z|^{2}}\right| z\right|^{-\alpha / 2}\right|^{2} d v(z) \\
\leq \int_{C^{n}}\left(\int_{C^{n}} H_{\alpha}^{2}(w, z) d v(w) \int_{C^{n}} H_{\alpha}^{2}(w, z)\right. \\
\left.\cdot\left|(f)_{\alpha / 2}^{+}(w)\right|^{2} \frac{e^{-|w|^{2}}}{|w|^{\alpha}} d v(w)\right) d v(z),
\end{gathered}
$$

where $H_{\alpha}^{2}(w, z)=e^{-(1 / 2)|z|^{2}}|z|^{-\alpha / 2}\left|\left(K_{z}^{\alpha}\right)_{\alpha / 2}^{+}(w)\right| e^{-(1 / 2)|w|^{2}}$ $\cdot|w|^{-\alpha / 2} \exp (\operatorname{Re}(q(z)-\overline{q(w)}))$.

If we can affirm both

$$
\begin{aligned}
& \sup _{z \in \mathbb{C}^{n}} \int_{C^{n}} H_{\alpha}^{1}(z, w) d v(w)<\infty, \\
& \sup _{z \in \mathbb{C}^{n}} \int_{C^{n}} H_{\alpha}^{2}(z, w) d v(w)<\infty,
\end{aligned}
$$

we would finish the proof because

$$
\begin{aligned}
\left\|T_{u}^{\alpha} T_{\bar{v}}^{\alpha} f\right\|_{F_{\alpha}^{2}}^{2} \leqslant & \int_{C^{n}}\left|(f)_{\alpha / 2}^{-}(w)\right|^{2} e^{-|w|^{2}} d v(w) \\
& +\int_{C^{n}}\left|(f)_{\alpha / 2}^{+}(w)\right|^{2} \frac{e^{-|w|^{2}}}{|w|^{\alpha}} d v(w) .
\end{aligned}
$$

To the finish, in terms of Lemma 1 and transformation, we can assert that

$$
\begin{aligned}
& \sup _{z \in \mathbb{C}^{n}} \int_{\mathbb{C}^{n}} H_{\alpha}^{1}(z, w) d v(w) \\
& \quad \leq \sup _{z \in \mathbb{C}^{n}} \int_{C^{n}}(1+|w \cdot \bar{z}|)^{\alpha / 2} e^{-(1 / 8)|z-w|^{2}}\left|e^{q(z-w)}\right| d v(w) \\
& \quad<\infty,
\end{aligned}
$$

$$
\begin{aligned}
& \sup _{z \in \mathbb{C}^{n}} \int_{C^{n}} H_{\alpha}^{2}(z, w) d v(w) \\
& \quad \leq \sup _{z \in \mathbb{C}^{n}} \int_{C^{n}} \frac{|z|^{-\alpha / 2}|w|^{-\alpha / 2} e^{-(1 / 8)|z-w|^{2}}}{(1+|z \cdot w|)^{-\alpha / 2}}\left|e^{q(z-w)}\right| d v(w) \\
& \quad<\infty .
\end{aligned}
$$

This implies that $\left\|T_{u} T_{\bar{v}} f\right\|_{\widetilde{F}_{\alpha}^{2}}$ is bounded and completes the proof.

Theorem $6((1) \Rightarrow(3))$. If $u$ and $v$ are two functions in the Fock-Sobolev type space $F_{\alpha}^{2}$, not identically zero, such that the operator $T_{u}^{\alpha} T_{\bar{v}}^{\alpha}$ is bounded on $F_{\alpha}^{2}$, then $\widetilde{|u|^{2}}(z) \widetilde{|v|^{2}}(z)$ is a bounded function on the complex space.

Proof. We omit the proof here for it is analogous to Theorem 2.6 in [12].

Theorem $7((3) \Rightarrow(2))$. Suppose that $u$ and $v$ are two functions in the Fock-Sobolev type space $F_{\alpha}^{2}$, not identically zero, such that $\widetilde{|u|^{2}}(z) \widetilde{|v|^{2}}(z)$ is bounded on $\mathbb{C}^{n}$. Then there is a complex linear polynomial $q(z)$ on $\mathbb{C}^{n}$ satisfying $u=e^{q}$ and $v=C e^{-q}$, where $C$ is a nonzero complex constant.

Proof. Now we only consider the case of $\alpha>0$ while the other case would be referred to in Theorem 2.7 in [12].

It is easy to see that, for any $u \in F_{\alpha}^{2}, \widetilde{u}(z)=\left\langle T_{u}^{\alpha} k_{z}^{\alpha}, k_{z}^{\alpha}\right\rangle_{\alpha}=$ $u(z)$. When $\alpha>0$, we use the triangle inequality and Hölder's inequality to calculate

$$
\begin{aligned}
|u(z)|^{2} \lesssim & \int_{C^{n}}\left|u(w)\left(k_{z}^{\alpha}\right)_{\alpha / 2}^{-}(w)\right|^{2} e^{-|w|^{2}} d v(w) \\
& \cdot \int_{C^{n}}\left|\left(k_{z}^{\alpha}\right)_{\alpha / 2}^{-}(w)\right|^{2} e^{-|w|^{2}} d v(w) \\
& +\int_{C^{n}}\left|u(w)\left(k_{z}^{\alpha}\right)_{\alpha / 2}^{+}(w)\right|^{2} \frac{e^{-|w|^{2}}}{|w|^{\alpha}} d v(w) \\
& \cdot \int_{C^{n}}\left|\left(k_{z}^{\alpha}\right)_{\alpha / 2}^{+}(w)\right|^{2} \frac{e^{-|w|^{2}}}{|w|^{\alpha}} d v(w) .
\end{aligned}
$$

Because $k_{z}^{\alpha}$ is a unit element, that is,

$$
\begin{aligned}
\left\|k_{z}^{\alpha}\right\|_{\widetilde{F}_{\alpha}^{2}}= & \left(\int_{C^{n}}\left|\left(k_{z}^{\alpha}\right)_{\alpha / 2}^{-}(w)\right|^{2} e^{-|w|^{2}} d v(w)\right)^{1 / 2} \\
& +\left(\int_{C^{n}}\left|\left(k_{z}^{\alpha}\right)_{\alpha / 2}^{+}(w)\right|^{2} \frac{e^{-|w|^{2}}}{|w|^{\alpha}} d v(w)\right)^{1 / 2},
\end{aligned}
$$

we can see that

$$
\begin{aligned}
& \int_{C^{n}}\left|\left(k_{z}^{\alpha}\right)_{\alpha / 2}^{-}(w)\right|^{2} e^{-|w|^{2}} d v(w) \lesssim 1, \\
& \int_{C^{n}}\left|\left(k_{z}^{\alpha}\right)_{\alpha / 2}^{+}(w)\right|^{2} \frac{e^{-|w|^{2}}}{|w|^{\alpha}} d v(w) \lesssim 1
\end{aligned}
$$


From the above inequations, the estimate of $|u(z)|^{2}$ turns into

$$
\begin{aligned}
|u(z)|^{2} \lesssim & \int_{C^{n}}|u(w)|^{2}\left|\left(k_{z}^{\alpha}\right)_{\alpha / 2}^{-}(w)\right|^{2} e^{-|w|^{2}} d v(w) \\
& +\int_{C^{n}}|u(w)|^{2}\left|\left(k_{z}^{\alpha}\right)_{\alpha / 2}^{+}(w)\right|^{2} \frac{e^{-|w|^{2}}}{|w|^{\alpha}} d v(w) \\
= & \widetilde{|u|^{2}}(z) .
\end{aligned}
$$

If $\widetilde{|u|^{2}}(z) \widetilde{|v|^{2}}(z)$ is a bounded function on $\mathbb{C}^{n}, \widetilde{|v|^{2}}(z)|u|^{2}(z)$ and $|u|^{2}(z)|v|^{2}(z)$ are both bounded on $\mathbb{C}^{n}$. By Liouville's theorem, the boundedness of $|u|^{2}(z)|v|^{2}(z)$ implies that there exists a constant $C$ such that $u v=C$. Since neither $u$ nor $v$ is identically zero, we have $C \neq 0$. That is, both $u$ and $v$ are nonvanishing. By Lemma 1 , there exists a complex polynomial $q(z)$ on $\mathbb{C}^{n}$ with $\operatorname{deg}(q) \leq 2$ such that $u=e^{q}$ and $v=C e^{-q}$.

On the other side, by the definition of Berezin transformation in this case,

$$
\begin{aligned}
& \widetilde{|u|^{2}}(z)|v|^{2}(z) \\
& =\int_{C^{n}}\left|u(w) \bar{v}(z)\left(k_{z}^{\alpha}\right)_{\alpha / 2}^{-}(w)\right|^{2} e^{-|w|^{2}} d v(w) \\
& \quad+\int_{C^{n}}\left|u(w) \bar{v}(z)\left(k_{z}^{\alpha}\right)_{\alpha / 2}^{+}(w)\right|^{2} \frac{e^{-|w|^{2}}}{|w|^{\alpha}} d v(w) .
\end{aligned}
$$

Now giving a sufficiently small $\delta>0$, we further obtain that

$$
\begin{aligned}
& \widetilde{|u|^{2}}(z)|v|^{2}(z) \gtrsim \int_{|w|>\delta}\left|u(w) \bar{v}(z)\left(k_{z}^{\alpha}\right)_{\alpha / 2}^{-}(w)\right|^{2} \\
& \cdot e^{-|w|^{2}} d v(w)+\int_{|w|>\delta}\left|u(w) \bar{v}(z)\left(k_{z}^{\alpha}\right)_{\alpha / 2}^{+}(w)\right|^{2} \\
& \cdot \frac{e^{-|w|^{2}}}{|w|^{\alpha}} d v(w) \gtrsim \int_{|w|>\delta}\left|u(w) \bar{v}(z) k_{z}^{\alpha}(w)\right|^{2} \\
& \cdot \frac{e^{-|w|^{2}}}{|w|^{\alpha}} d v(w) \\
& \gtrsim \int_{|w|>\delta}\left|(1+|z|)^{-\alpha / 2} e^{-(1 / 2)|z|^{2}} K_{z}^{\alpha}(w) e^{-(1 / 2)|w|^{2}}\right|^{2} \\
& \cdot\left|\frac{e^{q(w)-q(z)}}{|w|^{\alpha / 2}}\right|^{2} d v(w) .
\end{aligned}
$$

When choosing a constant $\varepsilon>0$ satisfying Lemma 1 , we can see that

$$
\begin{aligned}
& \widetilde{|u|^{2}}(z)|v|^{2}(z) \gtrsim \int_{|z-w|<\varepsilon} \mid(1+|z|)^{-\alpha / 2} \\
& \left.\cdot e^{-(1 / 2)|z|^{2}} K_{z}^{\alpha}(w) e^{-(1 / 2)|w|^{2}}\right|^{2}\left|\frac{e^{q(w)-q(z)}}{|w|^{\alpha / 2}}\right|^{2} d v(w) \\
& +\int_{|z-w| \geq \varepsilon}\left|(1+|z|)^{-\alpha / 2} e^{-(1 / 2)|z|^{2}} K_{z}^{\alpha}(w) e^{-(1 / 2)|w|^{2}}\right|^{2} \\
& \cdot\left|\frac{e^{q(w)-q(z)}}{|w|^{\alpha / 2}}\right|^{2} d v(w) .
\end{aligned}
$$

Using the similar method like the case $\alpha<0$, we can get the desired results and the proof is finished at this moment.

\section{Conclusions}

In this content, we deal with the Sarason's problem on the Fock-Sobolev type spaces and have a complete solution that $u=e^{q}, v=C e^{-q}$, where $q$ is a linear complex polynomial and $C$ is a nonzero constant. As stated in [1], we know that the Fock-Sobolev type space $F_{\alpha}^{2}$ clearly does not fall under the class of weighted Fock spaces $F_{\varphi}^{2}$. Therefore the Sarason's problem of weighted Fock spaces $F_{\varphi}^{2}$ is still open. We will focus on this open problem in the future study.

\section{Conflicts of Interest}

The authors declare that they have no conflicts of interest.

\section{Authors' Contributions}

All authors contributed equally. All authors read and approved the final manuscript.

\section{Acknowledgments}

This paper is supported by National Natural Science Foundation of China (Grants nos. 11471084 and 11301101), Young Innovative Talent Project of Department of Education of Guangdong Province (no. 2017KQNCX220), and the Natural Research Project of Zhaoqing University (nos. 201732 and 221622).

\section{References}

[1] H. R. Cho, J. Isralowitz, and J.-C. Joo, "Toeplitz Operators on Fock-Sobolev Type Spaces," Integral Equations and Operator Theory, vol. 82, no. 1, 2015.

[2] H. R. Cho, B. R. Choe, and H. Koo, "Fock-Sobolev spaces of fractional order," Potential Analysis, vol. 43, no. 2, pp. 199-240, 2015.

[3] D. Sarason, "Products of Toeplitz operators," in Linear and Complex Analysis Problem Book 3, V. P. Khavin and N. K. Nikolski, Eds., vol. 1573 of Lecture Notes in Math, pp. 318-319, Springer, Berlin, Germany, 1994.

[4] D. Zheng, "The distribution function inequality and products of Toeplitz operators and Hankel operators," Journal of Functional Analysis, vol. 138, no. 2, pp. 477-501, 1996.

[5] J.-D. Park, "Bounded toeplitz products on the bergman space of the unit ball in $\mathbb{C}^{n}$," Integral Equations and Operator Theory, vol. 54, no. 4, pp. 571-584, 2006.

[6] K. Stroethoff and D. Zheng, "Products of Hankel and Toeplitz Operators on the Bergman Space," Journal of Functional Analysis, vol. 169, no. 1, pp. 289-313, 1999.

[7] K. Stroethoff and D. Zheng, "Bounded Toeplitz products on the Bergman space of the polydisk," Journal of Mathematical Analysis and Applications, vol. 278, no. 1, pp. 125-135, 2003.

[8] K. Stroethoff and D. Zheng, "Bounded Toeplitz products on Bergman spaces of the unit ball," Journal of Mathematical Analysis and Applications, vol. 325, no. 1, pp. 114-129, 2007. 
[9] A. Aleman, S. Pott, and M. C. Reguera, "Sarason's conjecture on the Bergman space," International Mathematics Research Notices, pp. 1-30, 2016.

[10] F. Nazarov, A counterexample to Sarason's conjecture, preprint, available at: http://users.math.msu.edu/users/fedja/prepr.html, 1997.

[11] H. R. Cho, J.-D. Park, and K. Zhu, "Products of Toeplitz operators on the fock space," Proceedings of the American Mathematical Society, vol. 142, no. 7, pp. 2483-2489, 2014.

[12] J. J. Chen, X. F. Wang, J. Xia, and G. F. Cao, "Sarason's Toeplitz product problem on the Fock-Sobolev space," Acta Mathematica Sinica, pp. 1-9, 2017, https://doi.org/10.1007/s10114-017-5780-8.

[13] X. Wang, G. Cao, and J. Xia, "Toeplitz operators on FockSobolev spaces with positive measure symbols," Science China Mathematics, vol. 57, no. 7, pp. 1443-1462, 2014.

[14] H. Bommier-Hato, E. H. Youssfi, and K. Zhu, "Sarason's Toeplitz product problem for a class of Fock spaces," Bulletin des Sciences Mathématiques, vol. 141, no. 5, pp. 408-442, 2017.

[15] K. Seip and E. H. Youssfi, "Hankel operators on Fock spaces and related Bergman kernel estimates," The Journal of Geometric Analysis, vol. 23, no. 1, pp. 170-201, 2013.

[16] X. Wang, G. Cao, and K. Zhu, "BMO and Hankel Operators on Fock-Type Spaces," The Journal of Geometric Analysis, vol. 25, no. 3, pp. 1650-1665, 2015. 


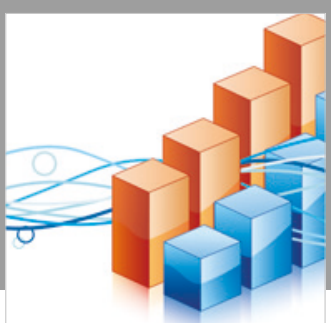

Advances in

Operations Research

\section{-n-m}
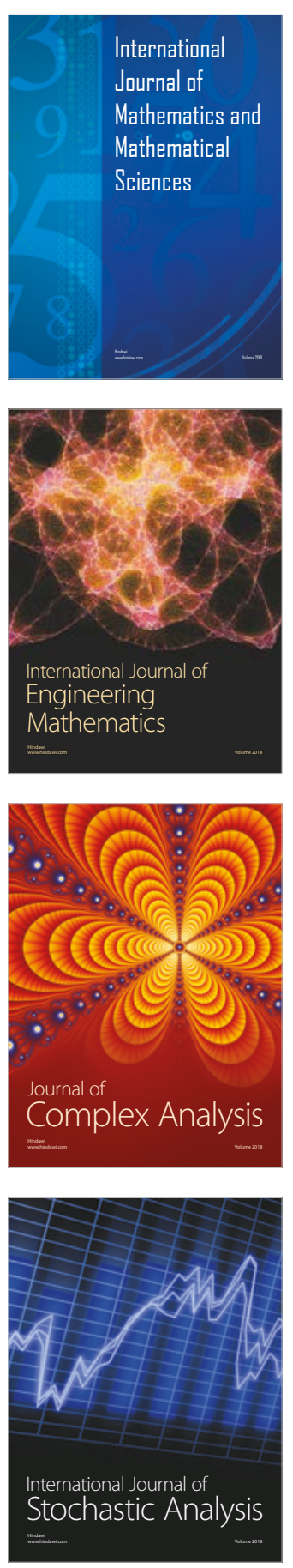
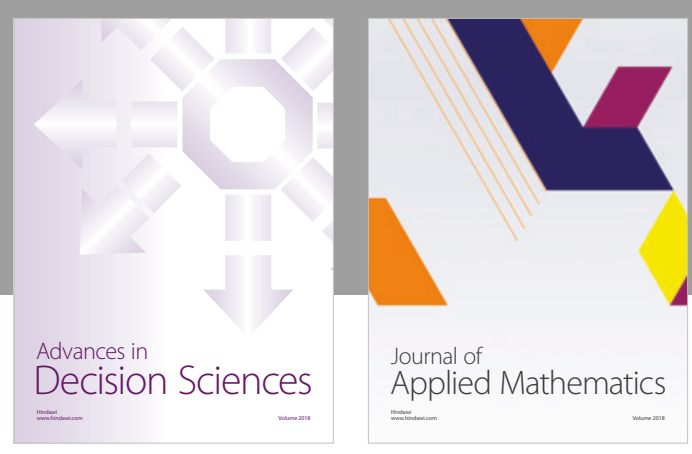

Journal of

Applied Mathematics
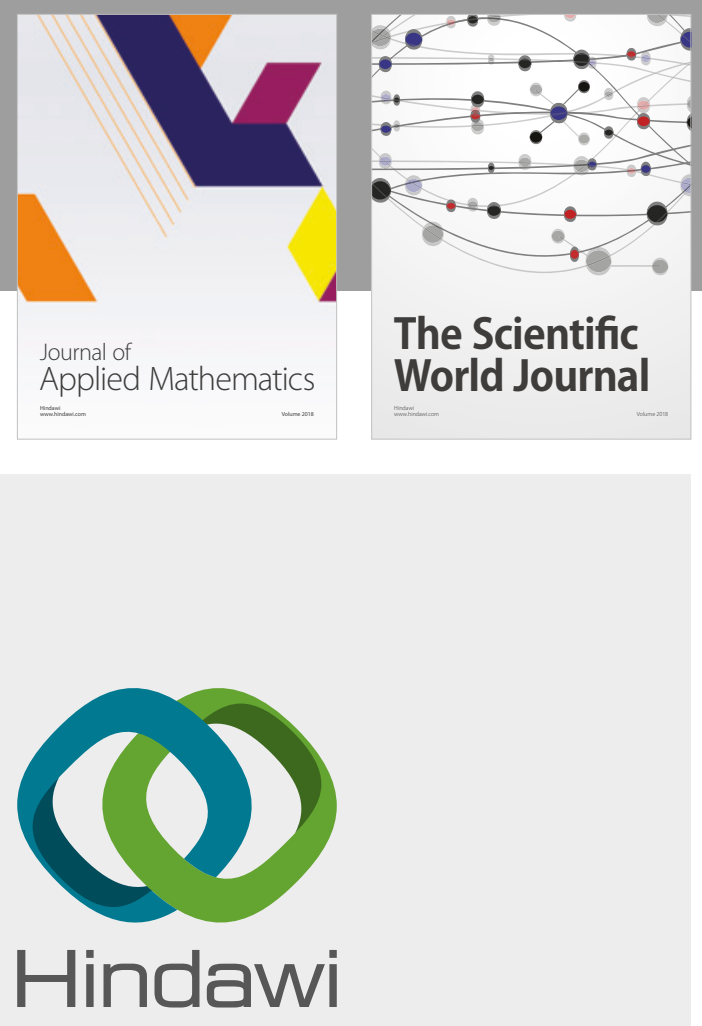

Submit your manuscripts at

www.hindawi.com

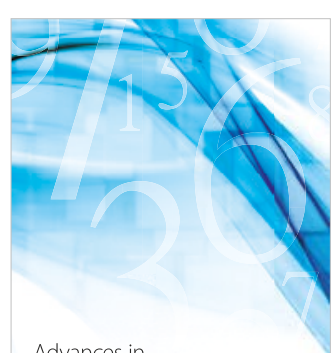

Advances in
Numerical Analysis
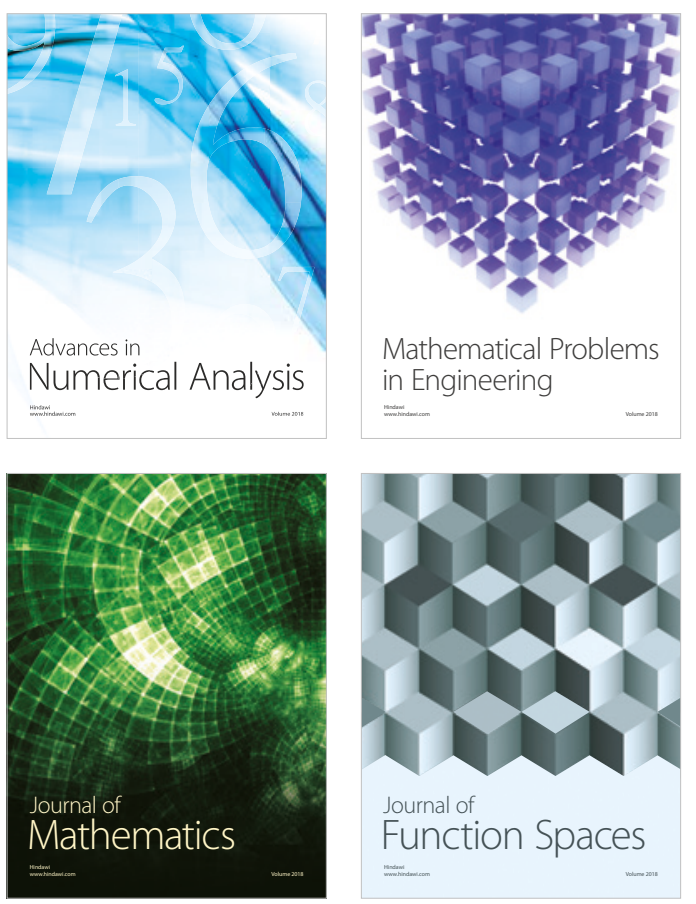

Mathematical Problems in Engineering

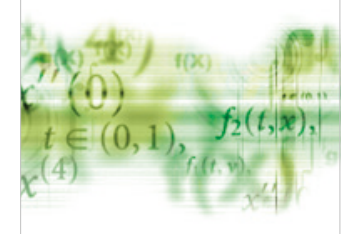

International Journal of

Differential Equations

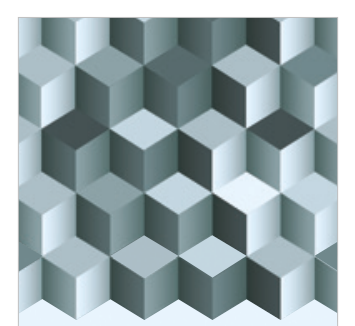

Journal of

Function Spaces

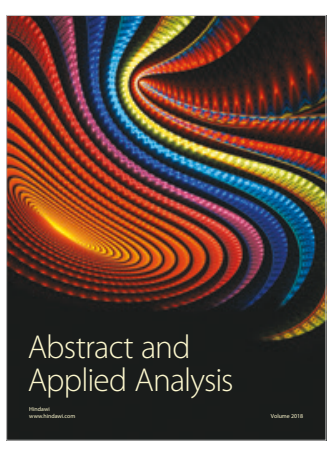

The Scientific

World Journal

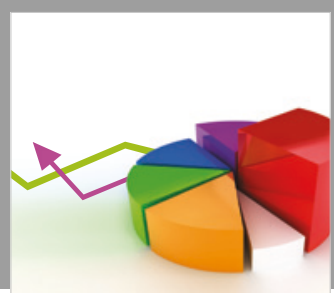

Journal of

Probability and Statistics
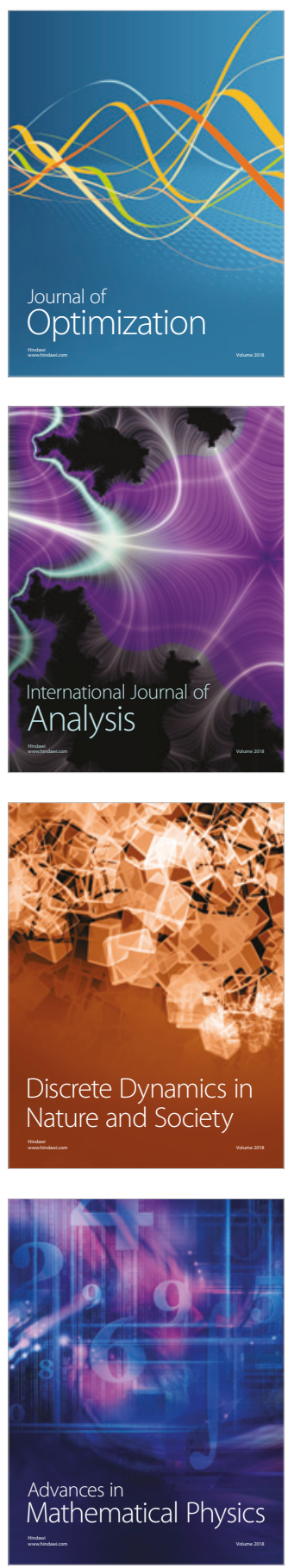